\title{
Protée
}

\section{Fin de l'histoire sans fin (Le Pays des eaux de Graham Swift)}

\section{Jean-François Chassay}

Volume 27, numéro 3, 1999

L'imaginaire de la fin

URI : https://id.erudit.org/iderudit/030573ar

DOI : https://doi.org/10.7202/030573ar

Aller au sommaire du numéro

Éditeur(s)

Département des arts et lettres - Université du Québec à Chicoutimi

ISSN

0300-3523 (imprimé)

1708-2307 (numérique)

Découvrir la revue

Citer cet article

Chassay, J.-F. (1999). Fin de l'histoire sans fin (Le Pays des eaux de Graham Swift). Protée, 27(3), 83-92. https://doi.org/10.7202/030573ar

\section{Résumé de l'article}

Cet article porte sur un ouvrage du romancier britannique Graham Swift, $L e$ Pays des eaux (Waterland), dans lequel l'importance de la fin est indissociable d'une constante ambiguïté, où les identités troubles sont sans cesse liées à la question de l'origine. Le narrateur, professeur d'histoire, délaisse les méthodes habituelles de sa discipline pour raconter sa propre vie à ses étudiants, reprenant la manière ancestrale du conte de fées. À l'histoire d'une vieille famille anglaise maintenant en pleine déliquescence se mêle une réflexion sur le développement des événements historiques et leur constante teneur catastrophique, dont l'épicentre serait la Deuxième Guerre mondiale. L'article examine comment la mise en texte de l'Histoire provoque une crise du sujet. 


\title{
FIN DE L'HISTO IRE SAN S FIN (LE PAYS DES EAUX DE GRAHAM SWIFT)
}

\author{
JEAN - FRAN ÇOIS CHASSAY \\ Le passé n'est jamais mort, il n'est même pas passé. \\ William Faulkner \\ "Tout est douleur» - la formule bouddhique, modernisée, serait: \\ "Tout est cauchemar».
}

Cioran

Aussi laissez-moi vous en raconter une autre. Laissez-moi vous raconter.

Graham Swift

Il était une fois une histoire qui ne pouvait finir, parce qu'elle ne parvenait à vivre que de sa propre fin, éternellement. Il était une fois une jeune fille de seize ans, obligée d'aller voir une vieille sorcière à cause d'un avortement ayant mal tourné; un professeur d'histoire qui abandonne la Révolution française pour s'enfoncer dans celle du langage, de son langage, de sa propre vie; un directeur d'école voulant abolir la discipline historique, parce qu'elle ne sert à rien selon lui; une Première Guerre mondiale qui semble signifier la fin de la civilisation, avant qu'on ne se rende compte, grâce à une Seconde Guerre, qu'il reste encore des choses à détruire; une industrie de la bière en pleine déliquescence; un pays des eaux noyé sous les inondations, revivant le déluge; une femme d'une cinquantaine d'années qui justifie l'enlèvement d'un bébé en affirmant qu'il s'agit d'un ordre lui venant de Dieu; une famille n'en finissant plus de dégénérer, de disparaître. Il était une fois l'histoire, mot par lequel il faut entendre la fin des temps, la mort, la folie. Il était une fois une narration romanesque ne parvenant plus à se raconter de manière linéaire et causale, malgré les événements historiques en amont de nos vies, parce que la mort et la folie ne se racontent pas comme ça. Parce que la mort, la folie, constitue un "Ici-maintenant», un atroce présent évoquant cette chose étrange (car, oui, on peut l'appeler "chose») sur laquelle il faut sans cesse insister: la réalité. Partout la fin, le désert à l'horizon de ce pays des eaux, des fins sans fin. Point final. 


\section{LE CONTE DE FÉES REMIS EN QUESTION}

Le Pays des eaux de Graham Swift se présente dès les premières lignes comme un conte de fées. Après avoir rappelé des propos revenant fréquemment dans la bouche de son père, le narrateur, Tom Crick, note qu'il s'agissait de "mots de contes de fées; un conseil de conte de fées. Mais c'était un lieu féerique que nous habitions. Une maison d'éclusier, au bord d'une rivière, au milieu des Fens. Loin du vaste monde»1. Cet univers qu'il nommera, un peu plus loin, «Un pays de conte de fées, après tout» (p.15), laisse penser que les mots collent au lieu, que le discours tenu dans cette contrée adopte les contours du paysage.

Pourtant, le rapport du narrateur au conte (et particulièrement au conte de fées) se révèle trouble, ambigu; les liens entre ce dernier et le mode d'énonciation dans Le Pays des eaux sont d'une complexité similaire à celle unissant le narrateur à ses origines, une lointaine lignée se perdant dans la nuit des Fens qui le poussera malaisément à devenir historien, pour contourner ses antécédents familiaux sans s'en éloigner vraiment. La relation entre le conte de fées et la discipline qu'il va pratiquer est d'ailleurs clairement établie:

Ma vocation de professeur d'histoire, voyez-vous, on peut directement l'attribuer aux histoires que ma mère me racontait lorsque j'étais enfant et que, comme la plupart des enfants, j'avais peur de l'obscurité. (p.90)

La révélation de ce passé perturbé - la crainte que ne surgisse de l'obscurité ce qui devrait rester caché risque toujours de voir s'effondrer comme un château de cartes l'équilibre précaire du narrateur. En conséquence celui-ci préfère l'esquive, esquive que le morcellement du texte de Swift, échappant à la linéarité, tend à redoubler. La structure formelle du Pays des eaux reporte sans cesse une chute qu'on sent inévitable dès les premières lignes, de la même façon que les circonvolutions des longues phrases du narrateur éloignent sans cesse les souvenirs dans les méandres d'événements présentés toujours de manière plus parcellaire. La crainte est grande d'arpenter «les champs de mines du passé» (p.433).
Il était une fois, donc, une histoire qui commence ainsi:

Au beau milieu d'une explication sur la façon dont, dans un bain de sang parisien, notre Monde Moderne avait commencé, [Tom Crick] s'interrompt et se met à raconter... ces histoires.

Quelque chose à propos de la vie au bord d'une rivière, quelque chose à propos d'un père qui attrapait des anguilles, et du corps d'un noyé trouvé dans la rivière, il y a des années de cela. (p. 18-19)

À partir de là, le lecteur apprendra, dans un ordre d'une complexité ne ressemblant en rien à ce que j'énonce ici un peu platement, la mort du jeune Freddie Parr, retrouvé noyé le 25 juillet $1943^{2}$, dans les eaux du pays de Fens, là où le père du narrateur est éclusier. Puis que cette mort n'est pas accidentelle comme on le croit, mais plutôt le résultat d'un meurtre: le frère aîné de Tom, Dick, a tué l'adolescent en le frappant d'un coup de bouteille à la tempe, avant de le précipiter à l'eau, le croyant responsable de la grossesse de Mary. Tom est pourtant le vrai géniteur. En essayant d'avorter - elle se précipite d'une hauteur d'environ un mètre cinquante, les jambes écartées, recommençant jusqu'à épuisement -, Mary se blesse gravement et demande l'aide d'une vieille femme à qui on attribue des pouvoirs maléfiques. Celle-ci ne fait qu'envenimer son état et l'adolescente finit par apprendre qu'elle ne pourra plus procréer. Elle épouse malgré tout Tom quelques années plus tard, mais devient folle au début de la cinquantaine, enlevant un bébé, convaincu que celui-ci lui a été désigné par Dieu comme le sien. Tom Crick voit sa carrière se terminer au moment où sa vie familiale est brisée, et à cause de cela en partie: comment laisser un homme enseigner à des adolescents alors que sa propre femme kidnappe des enfants?

Dick, des années auparavant, considérait également que les enfants tombaient du ciel. Il pensait pouvoir en avoir un avec Mary, simplement en la prenant dans ses bras. Puisqu'il est attendu par son propre père comme un Messie, la croyance de Dick en l'Immaculée conception n'a-t-elle pas quelque chose de légitime? Plus prosaïquement, une telle réaction 
s'explique par ses déficiences intellectuelles: il est une «tête de patate», incapable de s'exprimer et d'écrire correctement. Tom apprendra grâce à la découverte de lettres que la naissance de son frère est le fruit des amours illégitimes de sa mère et de son grand-père. Enfant dégénéré, résultat d'un tabou transgressé, il provient d'une «lignée royale» en pleine décadence, celle de brasseurs de bières qui firent les beaux jours de la région. L'histoire de cette famille, longuement racontée dans le roman, perturbée par la folie et les morts violentes, et dont Tom Crick est l'ultime descendant, voit dans la démence de Mary le dernier avatar d'une chute qui dure depuis un siècle, faite de bruits et de fureurs ${ }^{3}$.

Contrairement au conte de fées, l'histoire de la famille de Tom Crick, professeur d'histoire de 53 ans poussé vers une retraite anticipée, finit mal. Au-delà de cette importante différence thématique, l'énonciation même du roman soulève des problèmes propres à l'étude du conte, genre, comme on le sait, né de la littérature orale. Or, dans ce roman divisé en 52 chapitres de longueurs variables (entre une et 56 pages), Tom Crick se trouve devant sa classe et parle à ses étudiants. Il raconte une histoire - ou la conte, car qui peut garantir la véracité des faits évoqués? Mais les glissements d'un chapitre à l'autre, la déstructuration du temps, dans ce roman où il s'agit pourtant de suivre le destin d'une lignée familiale, ne permettent pas toujours de savoir quand Crick s'exprime devant ses élèves et quand la narration est dirigée vers un narrataire moins explicite. Dès lors, le narrataire extradiégétique, se confondant avec le lecteur virtuel, ne sait plus toujours distinguer les passages où le narrateur "parle» à ses étudiants (ou encore à Mary, ce qui se produit souvent), alors que les codes énonciatifs relèvent clairement de l'oralité, d'autres passages textuels où le récitatif se situerait davantage dans l'orbe de l'écrit (du journal personnel, par exemple).

Cette structure en soi évoque le passage du temps, alors que la narration est prise dans une tension entre l'oralité (un monde ancien, un monde du mythe "tout commença avec le premier conteur de la tribu» comme l'écrit Italo Calvino ${ }^{4}$ ) et l'écriture (un univers tourné vers la modernité). Non seulement cette structure rappelle le conte tout en le niant, mais elle impose une lecture de l'histoire qui s'interdit le fantasme consistant à totaliser les fragments en une interprétation globale et systémique. Pourtant, malgré les ruptures et le refus de la chronologie, il y a un fil conducteur qu'on peut retracer grâce aux codes mêmes du conte de fées, avec lequel le narrateur maintient pourtant une distance. ( Mais gardons-nous des contes de fées" [p.26] affirme-t-il, après avoir mentionné les racontars courant sur une femme accusée d'être une sorcière.)

"Il était une fois», formule consacrée ouvrant le conte, utilisée à l'envi par l'écrivain britannique dans son roman, prend ici une coloration particulière. «Il était une fois» exprime sémiotiquement, d'entrée de jeu, la possibilité quasi indéfinie pour le conte de fées de se répéter, de proposer un schéma dont Propp a su signaler la constance. Au contraire, on pourrait traduire la formule chez Swift de la manière suivante: "Il était une fois». C'est la singularité absolue de l'expérience qui compte. Les propriétés du conte de fées s'inversent et imposent les particularités suscitées par le parcours étonnant de la famille Crick, formant ainsi un véritable hapax au cœur de l'histoire, ramené à une catastrophe individuelle, unique. Pris en étau entre les catastrophes produites par l'évolution des nations et celle de sa propre famille, Crick vit une crise à la mesure du pays d'où il vient, les Fens, le waterland, terme constituant un véritable oxymore, monde de terre et d'eau à la fois, qui produit la vase dans laquelle on s'englue - «La vase: qui forme et mine par en dessous les continents; qui démolit en construisant; qui est simultanément alluvion et érosion; qui n'est ni progrès ni déclin» (p.22). Dans ce roman marqué par l'isotopie de l'eau, le verbe «sombrer» apparaît peut-être comme le terme clé du roman («Les Fens rétrécissent. Ils continuent à rétrécir aujourd'hui - et à sombrer ", p.27). Il s'agit d'un parcours paradoxal, hanté par le mythe, mais parallèle à une histoire politique dont on suit la tourmente.

Dès lors, ce double sens du syntagme figé «il était une fois» situe Crick à la frontière du conte 
(mythique) et de l'histoire, ou fait de cette dernière un mythe - un de ses étudiants ne s'écrie-t-il pas d'ailleurs "que l'histoire était un conte de fées" (p. 210)?

Le mythe d'origine débute, en nombre de cas, par une esquisse cosmogonique: le mythe rappelle brièvement les moments essentiels de la Création du Monde, pour raconter ensuite la généalogie de la famille royale, ou l'histoire tribale, ou l'histoire de l'origine des maladies et des remèdes, et ainsi de suite. Dans tous ces cas, les mythes d'origine prolongent et complètent le mythe cosmogonique. ${ }^{5}$

Or, les Fens exhibent un pays de début du monde, un décor «vaste et vide» qui, «dans leur nudité et leur vacuité se prêtent volontiers à l'imaginaire - et au surnaturel» (p.35). Cette terre d'élection verra naître et se développer la lignée royale d'un monde dégradé (celui de la bourgeoisie d'affaires), les Atkinson, brasseurs qui régneront en maître sur la région avant de s'effondrer, laissant au monde la jeune Mary, future épouse de Tom Crick, qui devient l'historiographe des deux familles.

Le mythe des commencements a quelque chose de bien séculier dans ce roman, dans la mesure où l'histoire de la famille Crick, qui est celle d'une résistance à l'eau ( «Ils s’identifièrent à la destinée des Fens, qui était de lutter, non pas pour, mais contre les eaux", p. 28), reprend la théorie de l'évolution. Ils sortent de l'eau pour conquérir la terre ${ }^{6}$. Pourtant, l'histoire naturelle sera rattrapée par l'histoire mythique selon un mode de confrontations génériques propre au roman de Swift.

On pourrait dire, écrit Eliade, que, pour les primitifs, la Fin $d u$ Monde a déjà eu lieu, bien qu'elle doive se reproduire dans un avenir plus ou moins éloigné. [...] Les mythes du Déluge sont les plus nombreux, et presque universellement connus (bien qu'extrêmement rares en Afrique). 7

L'inondation dans les Fens, après la Deuxième Guerre, véritable déluge qui ressemble à un châtiment, conduira à la mort du père de Tom Crick par broncho-pneumonie. Châtiment qui sonne presque le glas d'une famille, mais aussi d'un empire, deux ans après la fin de la guerre, à une époque où le rationnement existe toujours.

Et montrez-nous, s'il vous plaît, les fruits de notre victoire. [...]

Gandhi veut la restitution de son Inde. Ah, rêves! Ah, plans!

Voyez tout cela à travers la mémoire d'un homme de quarantehuit ans, né sous le règne de Victoria, blessé à Ypres, et sur le point de mourir de broncho-pneumonie. Qu'est-il advenu de cette sornette que nos grands-pères nous débitaient? (p.446)

La volonté de suivre les mésaventures de la famille parallèlement aux événements sociohistoriques ne conduit qu'à mettre davantage en lumière leur inévitable rencontre qui a tout de la fatalité du destin.

Le croisement de certaines formes génériques dans le roman accentue cette place accordée au mythe:

L'historiographie, la biographie et le roman historique sont issus

d'un ancêtre commun: l'épopée héroïque. Mais derrière cet ancêtre il y en a un plus éloigné, encore plus vénérable, premier ancêtre de toute narration, sinon de la communication humaine en général: le mythe. Et l'on dirait presque que l'historiographie aussi bien que la biographie et le roman historique continuent à devoir toute leur validité à cet héritage mythique, si parcimonieux qu'il puisse être devenu dans une longue suite d'héritages. ${ }^{8}$

Biographe et autobiographe, historiographe, historien qui réfléchit sur sa discipline, Tom Crick ne peut échapper au mythe. S'il serait oiseux de lire Le Pays des eaux comme un roman historique, on le voit néanmoins traversé de part en part par l'histoire, confrontant celle-ci à la fois à la narration et au réel:

Non, n'oubliez pas. N'effacez pas. Vous ne pouvez effacer. Mais

faitesen une histoire. Juste une histoire. Oui, tout est complètement fou. Qu'estce qui est réel? Une histoire. Rien qu'une histoire... (p. 299)

Si Crick raconte une épopée cependant, elle n'a rien d'héroïque. Elle se pense et s'énonce dans la négativité.

\section{L'HISTOIRE DÉSORIENTÉE}

Il est loin d'être innocent que Crick mette fin à l'histoire (à un enseignement traditionnel de la discipline) au moment où il cherche à parler de cette 
rupture fondamentale que fut, pour les Occidentaux, la Révolution française. Dans cette brèche entre l'ancien et le nouveau monde, Crick cherche à s'insérer, à retrouver l'origine des catastrophes de son existence à travers l'histoire, au moment où la crise de sa femme l'isole de sa propre vie, le coupe de ses propres repères. L'éclatement du récit est à la fois le signe d'une perte de contrôle, contrôle qu'il cherche à retrouver en balisant l'itinéraire de ses ancêtres de faits historiques, mais aussi de l'impossibilité de dire les choses en suivant un processus logique qui conduirait à démontrer trop clairement l'anéantissement impitoyable, en cette fin de vingtième siècle, d'une famille dont l'importance fut considérable. Devant l'impossibilité de dire l'indicible, Crick se réfugie dans la narration d'une histoire déjouée qui s'exprime selon des perspectives variées, cherchant à retrouver un sens parmi de multiples pistes «insensées», dominées par la folie, la violence, l'obsession du pouvoir. Cette crise a donc peu à voir avec la vision d'une fin de l'histoire à la manière d'un Francis Fukuyama qui, sur le modèle de la fin des idéologies, fantasme l'homogénéisation démocratique du monde sous le signe du libéralisme occidental (comme si cela ne participait pas de l'histoire, comme si le libéralisme n'était pas une idéologie $\left.{ }^{9}\right)$. Un des étudiants de Crick affirme: "La seule chose importante pour ce qui est de l'histoire, je pense, monsieur, c'est que celle-ci en est arrivée au point où il est probable qu'elle va bientôt finir" (p.20-21), mais derrière cette déclaration se cache l'idée eschatologique d'une fin prochaine de l'humanité. Cet étudiant sera d'ailleurs un des fondateurs, au collège, d'un "club de la fin du monde»:

Le club Holocauste. La ligue Anti-Armageddon. Nous n'avons

pas encore décidé du nom [...] Nous avons songé à lancer un magazine. Amener les gens à coucher leurs peurs par écrit. Comment ils voient la fin $d u$ monde, vous savez. Les dernières minutes, les dernières pensées, la panique, comment ce sera pour ceux qui ne s'en vont pas tout de suite. (p.313)

Ces discussions avec les étudiants exacerbent la crise vécue par Crick lui-même, pour qui tout s'enlise
- la vase, toujours - dans un présent où passé et futur s'engouffrent. Il dit lui-même:

Ainsi, oubliez, pour sûr, vos révolutions, vos tournants, vos grandes métamorphoses de l'histoire. Considérez plutôt le lent processus ardu, l'interminable processus ambigu: le processus de l'envasement humain - de l'assèchement du sol. (p.24)

Les processus historique et géologique, associés comme deux systèmes isomorphes, se conjuguent pour donner plus de force à un présent, celui de Crick, qui cherche à se jouer de la temporalité. Le passé n'est pas le passé, mais s'enracine dans le présent, selon un modèle assez semblable à celui qu'Hannah Arendt a déjà développé:

[L]e passé, dont la portée s'étend jusqu'à l'origine, ne tire pas en arrière mais pousse en avant, et c'est contrairement à ce que l'on attendrait, le futur qui nous repousse dans le passé. Du point de vue de l'homme, qui vit toujours dans l'intervalle entre le passé et le futur, le temps n'est pas un continuum, un flux ininterrompu; il est brisé au milieu, au point où «il» se tient; et «son» lieu n'est pas le présent tel que nous le comprenons habituellement mais plutôt une brèche dans le temps que «son» constant combat, «sa" résistance au passé et au futur fait exister. 10

Considérons par exemple la façon dont Crick développe ses hypothèses sur la Révolution française. Ce grand bond en avant manifeste selon lui

[...] l'idée d'un retour. Une rédemption; une restauration. Une réaffirmation de ce qui est pur et fondamental contre ce qui est décadent et faux. Un retour à un nouveau commencement... (p. 189)

Et ces messies révolutionnaires [...] avaient-ils réellement en tête une Société de l'Avenir? Pas la plus petite idée. Leur modèle était une Rome antique idéalisée. [...] Nos héros des temps nouveaux [...] brûlaient, eux aussi, de revenir en arrière. (p. 190)

Il faut en conclure que l'histoire commence quand les choses vont mal, par des désastres, à travers une idéalisation, une mythification des échecs du passé, déshistoricisés justement. Braquer son regard sur le passé pour éviter d'avoir à concevoir l'avenir serait une façon d'oublier les contingences du réel, d'oublier que 
«les faits humains sont rares, ils ne sont pas installés dans la plénitude de la raison, il y a du vide autour d'eux pour d'autres faits que notre sagesse ne devine pas; car ce qui est pourrait être autre» ${ }^{11}$. Mais en 1980, au moment où Crick raconte sa vie et celle de ses ancêtres, deux Guerres mondiales auront eu lieu et, après la Première, déjà, Valéry aura déclaré: «Nous autres, civilisations, nous savons maintenant que nous sommes mortelles». La théorie de l'évolution aura rattrapé celle des nations. Dorénavant, «l'histoire est l'enregistrement du déclin» (p. 193).

«Le temps n'est pas un continuum, un flux ininterrompu ", écrit Arendt. Personne ne le sait mieux qu'un historien d'aujourd'hui:

Cela va dans deux directions à la fois. Cela va en arrière en allant de l'avant. Cela fait une boucle. Cela emprunte des détours. N'allez pas tomber dans l'illusion que l'histoire est une colonne de marche bien disciplinée et infatigable qui s'enfonce sans jamais dévier dans l'avenir. [...] Mais comment savons-nous [...] que nous ne sommes pas en train de nous mouvoir dans un grand cercle? (p. 186)

Retour à un temps cyclique, à un temps mythique? Serions-nous dupes? Sommes-nous toujours enfermés dans ces «sociétés closes» sur lesquelles se penche Lukács pour parler des civilisations d'avant la Modernité? Traduisons autrement: il serait naif de croire que l'histoire sert à quelque chose (et cette absence d'utilité immédiate provoque par exemple chez le directeur du collège où enseigne Crick un mépris à l'égard de la discipline tout entière). Pour qu'elle serve, il faudrait qu'on puisse en dérouler le fil de manière linéaire, qu'on en déduise des rapports de causalité évidents suggérant une morale, une éthique. Or, l'histoire est une narration confuse, sans ordre, à partir de laquelle on ne peut tirer de principes, mais uniquement des indices. «Nous croyons voir un itinéraire obligé, un développement logique tendant vers un but. C'est l'illusion de la téléonomie, que de consacrer l'état actuel dans un processus évolutif, et d'interpréter le passé en fonction du présent» ${ }^{12}$. Cela, Crick en est bien conscient. C'est pourquoi il cherche plutôt les failles, les brèches dans le temps, ces indices qui le relieraient à «son» lieu, son présent. Son père, revenu des tranchées après la Première Guerre, complètement neurasthénique, pensait: «il n'y a que la réalité, il n'y a plus d'histoires» (p. 38). Quels sont ces instants surgissant comme des "Ici-maintenant", ces moments privilégiés qui ne se racontent pas mais se manifestent plutôt comme des seuils qu'on ne peut franchir, des passages du temps où le temps s'arrête et où on voit se cristalliser à distance, en amont, le présent dont nous sommes faits?

Combien de fois l'Ici-et-Maintenant vientil nous visiter? Cela advient si rarement que ce n'est jamais ce que nous imaginons, et c'est l'Ici-et-Maintenant qui se révèle être le conte de fées, non l'Histoire, dont la substance est à tout le moins déterminée à jamais et ne peut être changée. (p.88)

Au-delà du sens qu'elle peut avoir dans la diégèse, l'expression «ouvrir les écluses» s'entend évidemment ici dans un sens métaphorique. Si l'histoire s'incarne habituellement dans un espace, cet espace se révèle dans Le Pays des eaux toujours liquide, il glisse entre les doigts. Malgré la volonté d'assécher la terre des Fens, la liquéfaction s'impose toujours. Que faut-il retenir de tout ce qui se déverse, de ce flot continu, quelles sont les substances solides qui résistent à cet écoulement?

Soient deux scènes tirées du roman, déterminantes dans la chute de la famille Atkinson. Deux scènes qui présentent, littéralement, des chutes. En 1820, Thomas Atkinson est au faite de sa gloire. Sa brasserie emploie la plupart des travailleurs de la région et génère une véritable fortune. De plus, il vit avec une femme adorable. Mais, beaucoup plus vieux qu'elle, la jalousie s'empare de lui et le voilà inventant des histoires à son propos. Un soir où elle rentre tard, il la gifle - du moins est-ce ainsi que la rumeur évoque la scène, dans cet espace des Fens où on adore raconter des histoires -, et sous le choc elle tombe, se cogne la tête contre le coin d'un bureau et perd complètement l'esprit. Pendant les cinquante ans que dure encore sa vie, elle reste silencieuse, assise tout le jour dans un coin de sa chambre. Après sa mort, sur cette terre hantée par les superstitions, Sarah Atkinson devient 
une histoire, un conte noir: nombreux sont ceux qui affirment avoir aperçu son fantôme, toujours au moment où des événements tragiques se déroulent dans la région. Dans l'infinie variété des futurs possibles, ce micro-événement - un coup malheureux, une mauvaise chute, au mauvais endroit - transforme définitivement l'avenir de la famille. Moins que l'événement suivant cependant, qui place au centre de la tourmente Ernest Atkinson et se produit près d'un siècle plus tard, en 1911, sans qu'on puisse déterminer le poids exact de sa responsabilité dans la catastrophe.

Cette année-là, George V accède au trône. Pour fêter l'événement, Ernest Atkinson, qui doit redorer son image auprès de la population, propose une nouvelle marque, la Coronation ale, bière du couronnement dont les mille premières bouteilles sont livrées gratuitement. Que contenait-elle de particulier? Faut-il la rendre responsable de la véritable folie qui s'empare de la population pendant la nuit et provoque l'incendie de la brasserie? Est-ce Atkinson lui-même, profitant de la confusion, qui l'a déclenchée, comme certains le soupçonnent par la suite? Autant de questions auxquelles on ne peut proposer que des hypothèses de réponses. L'origine de l'événement, l'ordre et les raisons de son déroulement, importent moins que sa symbolique emblématique. "La violence est contingence pure, mais son stade ultime est d'imposer un sens» ${ }^{13}$. La chute de la grande cheminée de la brasserie est le signe idoine de la décadence de la famille Atkinson.

Lorsque à minuit (car ce fut la dernière heure qui s'inscrivit à jamais sur le cadran altier de sa pendule) la cheminée trembla, chancela et, en même temps que ses frises à l'italienne et ses aiguilles de pendules paralysées, sombra verticalement à une vitesse vertigineuse dans le cratère embrasé du corps de bâtiment, ce fut avec l'accompagnement d'une tonitruante ovation [...] (p. 236) 14

Que cette chute se produise à minuit précisément s'avère un signe révélateur. D'autant plus qu'il s'agit d'une horloge monumentale qui s'écroule, premier modèle historique, selon Jean-Claude Beaune, de l'automate.
À la limite du vivant et du mort, l'automate-horloge est la figure exemplaire du temps technique, l'instantané répétitif de son éternité. La fonction principale de l'automate est [de] retrouver la cohésion logique de l'univers et l'harmonie du vivant et $d u$ mort, du bruit et du silence. Objet scientifique et métaphysique, mythique et utopique, il est incitation exemplaire à retrouver ou promouvoir un ordre dans le chaos. Son ambiguïté relève $d u$ désir d'ordre et de la réalité irréductible du chaos. [...] Le chaos initial peut être regardé comme l'ordre originel; l'ordre construit artificiellement comme un chaos uniforme et mortel. 15

La destruction de cet automate temporel 16 marque symboliquement le retour à la dimension spécifiquement mythique autour de laquelle se construit l'histoire, un chaos originel auquel les «messies révolutionnaires» - voir 1789 , mais surtout 1793 - se réfèrent.

Comment interpréter l'histoire à travers ces deux micro-événements qui transforment de fond en comble l'univers social des Fens? Comme une aspiration, volontaire ou non, à faire l'histoire, à donner matière à raconter.

Il n'y a pas de hasard parce qu'il n'y a pas de sens, pas de raison de privilégier un moment particulier de cette histoire, ou cette histoire plutôt que telle autre. [...] Le hasard se dissout dans la douce indifférence du monde. 17

N'est-ce pas ce que déduit Crick lui-même, élargissant l'hypothèse à l'ensemble des événements politiques? Posez-vous la question: combien d'événements de l'Histoire se sont produits pour telle ou telle raison, mais fondamentalement pour aucune autre raison que le désir de faire en sorte que des choses arrivent? (p.62)

Ce qui signifie une volonté de voir se multiplier les catastrophes, "parce que la gaieté n'a jamais fait l'histoire" (p.131). Rien d'étonnant alors à ce que certains affirment avoir vu lors de l'incendie le spectre de Sarah Atkinson, celle qui annonçait la chute, un rictus sur le visage, au milieu des flammes. Rien d'étonnant non plus aux soupçons pesant sur Ernest Atkinson, figure du prophète prédisant les horreurs du XXe siècle. 


\section{UN DOUBLE DE CASSANDRE, UN MESSIE DÉGRADÉ}

Contrairement à son père et à son grand-père, représentant les Fens au parlement pour le Parti conservateur, Ernest Atkinson défend les couleurs de la gauche et échoue lamentablement, en 1910, à se faire élire.

Avant que [la Première Guerre] ne commençât, le père [Ernest] avait pris publiquement la parole contre la guerre, dont tous ressentaient la venue comme une aventure exaltante. Il dit aux gens que, lorsque celle-ci viendrait, ce serait une chose terrible, un vrai désastre. Mais les gens se gaussèrent et se moquèrent du père. (p.300-301)

Ce rôle de Cassandre politique, Atkinson l'assume et en paye le prix. Désabusé, résigné, il s'enferme avec sa fille dans un hôpital qu'il a fait construire pour aider les soldats blessés gravement, y compris psychologiquement, pendant la guerre. C'est là que la mère de Tom et fille d'Ernest, Helen Atkinson, devait rencontrer son mari.

Le destin tragique du grand-père de Tom Crick le conduira vers une mort violente. Cassandre est exécuté, Ernest Atkinson se suicide. Car s'il démontre que la divination n'est pas le privilège des dieux, sa prophétie demeure un échec. Sa vengeance prendra forme à travers la volonté de mettre au monde un Messie, un être à la mesure de ses aspirations. La catastrophe n'en sera que plus spectaculaire.

$\mathrm{Si}$ on peut voir Ernest Atkinson comme un double inversé de Cassandre - un Cassandre au masculin -, on constatera qu'il inverse un autre mythe, celui d'CEdipe, puisque dans ce cas c'est le père qui a une relation sexuelle avec sa fille. Et de cette relation devait naître un enfant, qui sera pour la loi celui d'Henry Crick et d'Helen Atkinson. Il est temps, pour conclure, d'en venir au personnage principal de ce roman, Dick Crick, l'enfant débile.

Ce fils désiré ne sera pas, aux dires de son père génétique, un enfant comme les autres:

- Et tu prendras soin de lui - de mon fils, je veux dire?

- Si c'est un fils.

- Et il sera le Sauveur du Monde?
- Il sera le Sauveur du Monde.

Ainsi le père et la fille tombèrent-ils d'accord. (p. 304-305)

La scène de l'accouchement prend place dans un lieu qui, sans être une étable, rappelle ironiquement, par la pauvreté dont elle est empreinte, la naissance du Christ.

Et c'est là, dans ce pavillon de bord d'écluse que, par une aube grise de mars, sous l'œil d'Ada Berry, la sage-femme d'Hockwell et d'Apton, est né l'enfant dont Ernest Atkinson souhaitait qu'il fût le Sauveur du Monde.

Et qui se révéla être une tête de patate. (p.306)

Le fils, conséquence de l'inceste, exprime physiquement les signes du déclin. Un Messie ne peut naître d'une lignée déchue, repliée sur elle-même et qui s'auto-engendre comme ces vieilles familles royales dégénérées, au sang débile. L'espoir d'un Sauveur repose chez Atkinson sur l'impression tenace qu'avec la Première Guerre le monde est définitivement entré dans l'orbe de la fin.

C'est de ces mythes d'une catastrophe finale, qui sera en même temps le signe annonciateur de l'imminente re-création $d u$ Monde, que sont sortis et se sont développés, de nos jours, les mouvements prophétiques et millénaristes des sociétés primitives. 18

Et pourtant, malgré l'échec manifeste, tout se cristallise autour de Dick, figure « du désenchantement inévitable qui suit une longue et vaine exaltation messianique ${ }^{19}$. Le Sauveur arrive dans un monde déchu et ressemble à une mauvaise blague. Voici comment il apparaît, dans une scène où Tom Crick décrit la réalité vécue par son père, au début des années trente:

[...] foyer fixe, flux des eaux, pays uniformément plat; femme magnifique. Et qui devint père de deux fils (nés en 1923 et 1927), dont le premier se révéla être un demi-crétin qui était amoureux de son vélomoteur. (p.206)

Cette scène, rassemblant un certain nombre de signes propres au conte de fées (métaphorisé dans un cadre de vie bourgeois) se termine abruptement par ce 
syntagme presque burlesque, si ce n'était son aspect tragique. Mais justement, cet aspect tragique donne tout son poids, sur le plan diégétique, au personnage de Dick, aboutissement et synthèse à sa façon des familles Crick et Atkinson.

[...] Il nous faut [...] la possibilité d'un cataclysme, de sorte que, quand une situation se révèle sans issue, nous puissions espérer le salut d'un messie, d'un héros conquérant, d'un deus ex machina ou de quelque autre agent ayant le pouvoir d'en finir avec l'insupportable, et d'instituer ce qui semblait jusquelà inaccessible. 20

Dick est à la fois le cataclysme et le Messie, la personnification du monstre contre lequel il faut lutter pour ne pas en devenir un soi-même, pour paraphraser Nietzsche, mais également le signe d'une exception, d'une différence, d'une figure singulière. Habile de ses mains (comme un fils de charpentier...), il travaille dans l'eau et sent perpétuellement la vase, comme s'il était issu de la terre elle-même, réactivant la figure du Golem, autre être mythique. Lors de la scène finale, alors qu'il disparaît dans des flots vaseux vers la fin de la guerre, le narrateur ne dit-il pas qu'il sent «l'odeur de quelque chose que l'on remonte des profondeurs primitives. L'odeur qui hante la chambre de Dick»? "Et l'odeur de vase est l'odeur du sanctuaire, est l'odeur de l'amnésie. Il est ici, il est maintenant. Il n’est pas là-bas ou tantôt. Ni passé ni avenir. [...] Et il est le sauveur du monde...» (p.466). "Il est ici, il est maintenant», bref il est «l'Ici-etmaintenant», le temps hors temps, le point de rupture, la résistance au passé et au futur.

Cette création messianique, ce roi attendu sur terre a été préparé de longue date. Car la Coronation ale, la bière de couronnement aux pouvoirs étonnants brassée par Ernest Atkinson, Dick en héritera. Une douzaine de bouteilles, dans un coffre, avec une lettre de son véritable père, qu'il boira pour l'essentiel peu de temps avant de se jeter dans l'eau, disparaissant à la vue de tous. Et lorsque son propre père se tue avant sa naissance, c'est après en avoir ingurgité une quantité considérable: «Il se mit à [boire] cet extraordinaire breuvage visionnaire que ceux qui savent boire devraient être seuls à boire - que seul un Sauveur du Monde sait véritablement boire» (p.310). Alcool pour prophète, alcool pour les Cassandre de ce monde, qui se déverse comme les eaux inarrêtables des Fens, comme ce flot circulant au hasard et qu'on ne parvient pas à contenir.

Malgré les malheurs familiaux qui s'acharnent sur lui, Tom Crick n'est pas dupe: le monde n'approche pas plus de sa fin qu'auparavant et son histoire catastrophique ne fait que s'épuiser dans la douce indifférence du monde.

Oui, la fin du monde est de nouveau sur le tapis - mais cette

fois c'est peut-être pour de bon. Mais ce sentiment n'est pas

nouveau. [...] C'est le vieux, vieux sentiment, que tout équivaut

peut-être à rien. (p.354-355)

Le roman de Swift propose une stratégie narrative évoquant les «récits de vie», mais en se servant d'une aventure singulière, celle de Tom Crick, pour raconter celle de tous les Crick et les Atkinson et embrasser le monde: les événements qui traversent, même à distance, l'épopée des deux familles. Il joue du récit de vie pour proposer quelque chose qui dépasse celui-ci tout en s'en inspirant, laissant bruire la rumeur du monde, permettant en fin de compte

[...] une pluralité de voix et d'instances énonciatrices et non une seule comme l'expression "énonciateur» au singulier risquerait de le laisser croire... L'instance de l'énonciation (l'énonciateur) et la stratégie énonciative lorsqu'on les reconstitue a posteriori et toujours hypothétiquement ne font pas apparaître une unité cohérente et non contradictoire [...] Le sujet parlant en tant qu'énonciateur apparaît divisé, traversé par des voix et des intentionnalités distinctes et souvent opposées. Il y a donc des énonciateurs et des énonciataires qui leur correspondent dans un discours. 21

La fin existe depuis toujours si on peut dire, un historien ne peut qu'en être conscient:

Il était une fois des gens qui croyaient à la fin du monde. [...] voyez combien de fois et sous combien de prétextes la fin $d u$ monde a été prophétisée et préuue, calculée et imaginée. Mais c'était là, bien sûr, de la superstition. Le monde a grandi. Il n'a pas connu de fin. (p.440-441) 
Il reste que la crise grave vécue par Tom Crick, alors que «sa femme n'est plus qu'une histoire», lui permet de voir avec plus d'acuité les signes autour de lui annonçant la déchéance, le déclin, la chute, qui signalent qu'on en revient toujours là, car l'histoire n'a rien à voir avec le bonheur. Le roman de Graham Swift actualise une affirmation d'Adorno:

[...] il n'y a plus maintenant de beauté et de consolation que dans le regard qui se tourne vers l'horrible, s'y confronte et maintient, avec une conscience entière de la négativité, la possibilité d'un monde meilleur. 22

\section{N O TES}

1. G. Swift, 1995: 13. Les références au roman renverront toutes à cette édition. La version anglaise a été utilisée pour vérifier l'authenticité des citations, le traducteur ayant une étrange tendance à ajouter parfois des phrases de son cru...

2. Pour l'anecdote, rappelons que la nuit du 25 au 26 juillet 1943 au cours de laquelle meurt Freddie Parr, événement qui est le véritable embrayeur narratif de ce roman, est communément appelée «la nuit mémorable». C'est la nuit pendant laquelle Mussolini est arrêté. Même délivré peu de temps après par les parachutistes allemands, il n'a plus de pouvoir. Sa chute est considérée à l'époque comme «le début de la fin» de la guerre. Un dictateur tombe, les autres suivront.

3. L'allusion à Faulkner (via Shakespeare) n'est pas gratuite.

L'influence de l'écrivain américain est indéniable et on peut voir par exemple en Dick Crick un double de Benjy. La parution du dernier roman de Swift, Last Order (La Dernière Tournée), gagnant du Booker Prize, a d'ailleurs donné lieu à une solide polémique, un critique ayant accusé ce roman d'être un plagiat de As I Lay Dying (Tandis que j'agonise). Pour utiliser une litote, disons que l'accusation était nettement exagérée.

4. I. Calvino, $1984: 11$.

5. M. Eliade, 1978: 51 .

6. Et Francis Crick, un des ancêtres de Tom, n'est-il pas l'homonyme d'un grand biologiste anglais dont les recherches furent déterminantes dans la découverte de l'ADN, et donc dans celles des théories sur l'hérédité?

7. M. Eliade, 1978: 71. Sur l'importance du déluge comme cataclysme cosmique, voir en particulier L. Boia, 1989.

8. H. Broch, 1985: 248.
9. F. Fukuyama, 1992. La version originale date de 1989. Les critiques furent nombreuses. Pour un point de vue récent (et assassin!), voir A. Mattelart, 1998: 353-354.

10. H. Arendt, $1992: 20-21$

11. P. Veyne, 1996: 386.

12. I. Ekeland, $1991: 69-70$

13. Ibid., p. 71.

14. Cette scène est suivie immédiatement, au chapitre suivant, d'une narration par Tom Crick devant ses étudiants de la chute de la Bastille. "Non, la signification de cette clinquante victoire ne réside pas dans ses résultats tangibles mais dans sa valeur symbolique» (p.241). Encore une fois, les parallèles entre l'histoire familiale et l'histoire politique sont clairement sous-entendus pour le lecteur.

15. J-C. Beaune, 1980 : 113-114. Le modèle par excellence de cette représentation du temps à travers l'horloge-automate reste sans aucun doute The Mask of the Red Death d'Edgar Allan Poe.

16. L'automate, selon Beaune, se définit de la façon suivante:

"L'automate est une machine porteuse du principe interne de son mouvement qui, en conséquence, garde inscrits en ses composants matériels ou ses actions, l'illusion, le rêve ou la feinte de la vie" (ibid., p.7).

17. I. Ekeland, $1991: 146$

18. M. Eliade, $1978: 78$

19. Ibid., p. 76.

20.S. J. Gould, 1998: 23.

21. C. Chabrol, 1983: 17. Cité par R. Robin, Le Naufrage du siècle, Montréal et Paris, XYZ éditeur et Berg international, 1995, p.61. $R$. Robin a raison à mon avis d'affirmer que cette hypothèse pose problème dans le cas des récits de vie, mais elle s'applique bien à un roman qui s'en inspire.

22. T. Adorno, $1983: 21-22$

\section{RÉfÉREN CES BIBLIO gRAPHIQ U ES}

ADORno, T. [1983]: Minima Moralia, Paris, Payot. ARENDT, H. [1972]: La Crise de la culture, Paris, Gallimard, coll. "Folio", 1992.

BEAUnE, J.-C. [1980] : L'Automate et ses mobiles, Paris, Flammarion.

BOIA, L. [1989]: La Fin du monde: une histoire sans fin, Paris, La

Découverte.

BROCH, H. [1966]: Création littéraire et connaissance, Paris, Gallimard, coll. «Tel», 1985.

CAlvino, I. [1984]: La Machine littérature, Paris, Seuil.

Chabrol, C. [1983]: «Psycho-socio-sémiotique, Récits de vie et

sciences sociales", Revue des sciences humaines, 191, 8-25.

EKeland, I. [1991] : Au hasard. La chance, la science et le monde, Paris, Seuil.

EliADE, M. [1963] : Aspects du mythe, Paris, Gallimard, coll. «Idées", 1978.

FukUYAMA, F. [1989]: La Fin de l'histoire et le dernier homme, Paris, Flammarion, 1992.

GOULD, S. J. [1998]: Millénium. Histoire naturelle et artificielle de l'an 2000, Paris, Seuil.

MattelarT, A. [1998] : Histoire de l'utopie planétaire, Paris, La

Découverte.

SwiFT, G. [1985]: Le Pays des eaux, Paris, Robert Laffont, 1995.

VEYNE, P. [1971]: Comment on écrit l'histoire, Paris, Seuil, coll. «Points», 1996. 